\title{
Application of inclusive probability theory to heavy ion-atom collisions
}

\author{
P. Kürpick, H.J. Lüdde`, W.-D. Sepp, B. Fricke \\ Universität Kassel, Fachbereich Physik, W-3500 Kassel, Federal Republic of Germany
}

Received: 21 May 1992

\begin{abstract}
Using the independent particle model as our basis we present a scheme to reduce the complexity and computational effort to calculate inclusive probabilities in many-electron collision system. As an example we present an application to $K-K$ charge transfer in collisions of $2.6 \mathrm{MeV} \mathrm{Ne} \mathrm{Ne}^{9+}$ on $\mathrm{Ne}$. We are able to give impact parameter-dependent probabilities for many-particle states which could lead to $K L L$-Auger electrons after collision and we compare with experimental values.
\end{abstract}

PACS: $31.20 ; 34.70$.

\section{Introduction}

Since the first theoretical investigations to comprehend ion-atom collisions within the independent particle model considerable efforts have been put into matching the many-particle aspect of the collision problem and to take the Pauli principle into account. Over the last two decades several authors have developed and enlarged the theory of many-particle-hole inclusive probabilities [1-5]. Within the semi classical approximation the collision problem asks for the solution of the many-electron time dependent Schrödinger equation. On the basis of the independent particle model (IPM) the effective many-particle Hamiltonian is approximated by a sum of single-particle Hamiltonians, thus reducing the electronic many-particle problem to a set of single-particle equations for all electrons in the collision system. After solving this set of effective single-particle equations the formalism of inclusive probabilities allows to calculate many-particle-hole probabilities in terms of single-particle amplitudes.

Experimentally coincidence measurements between Xrays of Auger-electrons and angle resolved projectiles yield detailed impact parameter dependent probabilities for final subset occupancies and vacancies. Measure-

\footnotetext{
* Permanent address: Universität Frankfurt, Institut für Theoretische Physik, Federal Republic of Germany
}

ments for $K$ - and multiple $L$-shell vacancy production are available for a large number of symmetric and asymmetric heavy collision systems [6-10]. Up to date the many-particle-hole inclusive probability theory has found only few applications to those complex collision systems because the formalism available in literature is too complex for systems with more than a few electrons. Thus comparing with complex experimental measurements one is often forced to use the single-particle model. The complexity of the formalism of inclusive probabilities is caused by the large number of determinants to be calculated and summed up. We present a scheme to reduce this complexity as well as the computational effort, thereby facilitating the application of inclusive probabilities to collision systems with a large number of electrons. We also present an application to the collision system $2.6 \mathrm{MeV}$ $\mathrm{Ne}^{9+}$ on $\mathrm{Ne}$ for which detailed coincidence measurements for $K-K$ charge transfer are available. We compare results performed within the single-particle model with calculations using the inclusive probability theory to match the full experimental questions including Auger-electron in the $L$-shell of projectile and target.

\section{Method}

First a brief resume of the formalism of inclusive probabilities, for a complete description see $[4,5]$ : when using the independent particle model with an effective Hamiltonian $H^{\text {eff }}$ the many-electron Schrödinger equation

$$
\begin{gathered}
\hat{H}^{\mathrm{eff}}\left(x_{1} \cdots x_{N}, t\right) \Psi\left(x_{1} \cdots x_{N}, t\right) \\
=\mathrm{i} \hbar \frac{\partial}{\partial t} \Psi\left(x_{1} \cdots x_{N}, t\right)
\end{gathered}
$$

reduces to a set of effective single-particle equations

$\hbar^{\mathrm{eff}} \psi_{j}(x, t)=\mathrm{i} \hbar \frac{\partial}{\partial t} \psi_{j}(x, t)$ 
which must be solved for all $j=1, \cdots, N$ electrons involved in the collision system.

The solutions of the single-particle equations 2 yield the many-particle solution $\left|i_{1} \cdots i_{N}, t_{f}\right\rangle$ as the latter is a determinant built-up from $\psi_{j}\left(x, t_{f}\right)$ of the former.

The exclusive transition probability $P_{f_{1} \cdots f_{N}}$ to a final configuration $\left|f_{1} \cdots f_{N}\right\rangle$ is given as a $N \times N$-determinant

$$
\begin{aligned}
P_{f_{1} \cdots f_{N}} & =\left|\left\langle i_{1} \cdots i_{N}, t_{f} \mid f_{1} \cdots f_{N}\right\rangle\right|^{2} \\
& =\operatorname{det}\left(\gamma_{n n^{\prime}}\right),
\end{aligned}
$$

where $\gamma_{n n^{\prime}}$ are the single-particle density matrix elements

$\gamma_{n n^{\prime}}=\sum_{j=1}^{N}\left\langle f_{n} \mid \psi_{j}\right\rangle\left\langle\psi_{j} \mid f_{n^{\prime}}\right\rangle$.

The inclusive probability $P_{f_{1}} \ldots f_{q} \Sigma$ of finding $q$ of $N$ electrons in the subconfiguration $\left|f_{1} \cdots f_{q}\right\rangle$ while the remaining $N-q$ electrons are not observed explicitly was shown to be given as a $q \times q$-determinant constructed from the single-particle density matrix $\gamma[5]$

$P_{f_{1} \cdots f_{q} \Sigma}=P_{f_{1} \cdots f_{q}}$.

Using the recursive relation

$$
P_{f_{1} \cdots f_{q}}=P_{f_{1} \ldots f_{4}, f_{q}+1}+P_{f_{1} \cdots f_{q}}^{f_{q}+1},
$$

with subscripts denoting occupancies and superscripts vacancies permits to calculate inclusive probabilities with occupancies as well as vacancies specified [5]. The inclusive probability $P_{f_{1}, \ldots, \cdots, f_{q}}^{f_{q}+1, \cdots}$ of finding the $q$ states $f_{1}, \cdots, f_{q}$ occupied and the complementary configuration $f_{q+1}, \cdots, f_{L}$ empty in the subset $\mathscr{L}$ of $L$ states can be expressed in terms of simple inclusive probabilities [5].

$$
\begin{aligned}
P_{f_{1}, \cdots, \cdots, f_{4}}^{f_{4}}= & P_{f_{1}, \cdots, f_{q}}-\sum_{f_{q}+1} P_{f_{1}, \cdots, f_{4}, f_{14}+1} \\
& +\sum_{f_{q}+1<f_{4+2}} P_{f_{1}, \cdots, f_{4}, f_{4}+1, f_{4}+2} \\
& \mp \cdots(-1)^{L-q} P_{f_{1}, \cdots, f_{4}, f_{4+1}, \cdots, f_{L}} .
\end{aligned}
$$

The probability $P_{f_{1}}, \ldots, \ldots, f_{q}, f_{L}$ is exclusive in the subset $\mathscr{L}$.

Final subset configurations are detected in available experimental measurements by measuring one or more $\mathrm{X}$-rays or Auger-electrons in coincidence with the angle resolved projectile. The experimental energetic resolution of X-rays resp. Auger-electrons merely defines whether the photon resp. electron comes from the $K$ - or $L$-shell but gives no information on the exact electronic configuration of the collision system. Therefore only a minimum number of vacancies or occupancies is measured and not the absolute number of vacancies or occupancies within a subset $\mathscr{L}$ of $L$ states. Matching the experimental question needs the calculation of the many-particle-hole inclusive probability $P_{q \supseteq q_{0}}^{L-q}$ to find at least $q_{0}$ occupancies or vacancies in the subset of $L$ states. $P_{q \geq q 0}^{L-q}$ is given as
the sum over all inclusive probabilities $P_{q}^{L} q-q$ to find $e x-$ actly $q=q_{0}, \cdots, L$ states occupied and $L-q$ states unoccupied because $P_{q}^{L-q}$ is exclusive within the subset $\mathscr{L}$.
$P_{q \geqq q_{0}}^{L-q}=\sum_{q=q_{0}}^{L} P_{q}^{L-q}$.

$P_{q}^{L-q}$ is given as an ordered sum over all probabilities $P_{f i}^{q}, \ldots, f_{4}, f_{L}$

$P_{q}^{L-q}=\sum_{f_{1}<\cdots<f_{4}} P_{f_{1}, \ldots, f_{q}}^{f_{q}+1, \cdots, f_{L}}$.

Using (8) we express $P_{q}^{L-q}$ in terms of simple inclusive probabilities.

$$
\begin{aligned}
& P_{q}^{L-q}=\sum_{f_{1}<\cdots<f_{q}}\left(P_{f_{1}, \cdots, f_{q}}-\sum_{f_{q}+1} P_{f_{1}, \cdots, f_{q}, f_{q}+1}\right. \\
& +\sum_{f_{4+1}<f_{4+2}} P_{f_{1}, \cdots, f_{4}, f_{4+1}, f_{4+2}} \\
& \left.\mp \cdots(-1)^{L-q} P_{f_{1}, \cdots, f_{q}, f_{q}+1, \cdots, f_{L}}\right) \text {, } \\
& P_{q}^{L-q}=\sum_{f_{1}<\cdots<f_{q}} P_{f_{1}, \cdots, f_{q}} \\
& -\sum_{f_{1}<\cdots<f_{4}} \sum_{f_{q}+1} P_{f_{1}, \cdots, f_{q}, f_{q+1}} \\
& +\sum_{f_{1}<\cdots<f_{q} f_{q+1}<f_{q}, 2} P_{f_{1}, \cdots, f_{q}, f_{q+1}, f_{q+2}}-\cdots \\
& =\sum_{f_{1}<\cdots<f_{q}} P_{f_{1}, \cdots, f_{q}} \\
& -(q+1) \sum_{f_{1}<\cdots<f_{q}<f_{q+1}} P_{f_{1}, \cdots, f_{q}, f_{q+1}} \\
& +\frac{(q+2)(q+1)}{2} \sum_{f_{1}<\cdots<f_{q}<f_{q}+1<f_{q}+2} \\
& \times P_{f_{1}, \cdots, f_{4}, f_{4}+1, f_{4+2}}-\cdots \\
& =\sum_{i=0}^{L-q}(-1)^{i}\left(\begin{array}{c}
q+i \\
i
\end{array}\right) \mathscr{P}_{q+i},
\end{aligned}
$$

with

$\mathscr{P}_{q+i}=\sum_{f_{1}<\cdots<f_{i+i}} P_{f_{i}, \cdots, f_{q+i}}$

Inserting (11) into (9) leads to

$P_{q \geqq q_{0}}^{L-q}=\sum_{q=q_{0}}^{L} P_{q}^{L-q}=\sum_{q=q_{0}}^{L} \sum_{i=0}^{L-q}(-1)^{i}\left(\begin{array}{c}q+i \\ i\end{array}\right) \mathscr{P}_{q+i}$,

$P_{q \geq q 0}^{L-q}$

$=\left(\begin{array}{c}q_{0} \\ 0\end{array}\right) \quad \mathscr{P}_{90}-\left(\begin{array}{c}q_{0}+1 \\ 1\end{array}\right) \mathscr{P}_{90+1}+\left(\begin{array}{c}q_{0}+2 \\ 2\end{array}\right) \mathscr{P}_{40+2}+\cdots$

$=\left(\begin{array}{c}q_{0}+1 \\ 0\end{array}\right) \mathscr{P}_{40+1}-\left(\begin{array}{c}q_{0}+2 \\ 1\end{array}\right) \mathscr{T}_{40+2}+\left(\begin{array}{c}q_{0}+3 \\ 2\end{array}\right) \mathscr{P}_{40+3}+\cdots$

$=\left(\begin{array}{c}q_{0}+2 \\ 0\end{array}\right) \mathscr{D}_{40+2}-\left(\begin{array}{c}q_{0}+3 \\ 1\end{array}\right) \mathscr{P}_{40+3}+\left(\begin{array}{c}q_{0}+4 \\ 2\end{array}\right) \mathscr{P}_{40+4}+\cdots$

$+\left(\begin{array}{l}L \\ 0\end{array}\right) \mathscr{P}_{L}$. 
Reordering the sum we get:

$P_{q \geqq q_{0}}^{L-q}=\sum_{k=0}^{L-q_{0}} \sum_{j=0}^{k}(-1)^{j}\left(\begin{array}{c}q_{0}+k \\ j\end{array}\right) \mathscr{P}_{q_{0}+k}$.

Starting with (9) and (10) the computational effort to calculate the probability $P_{q \geqq q_{0}}^{L-q}$ is reduced, i.e. each determinant $P_{f_{1}, \cdots, f_{q+i}}$ for a given combination of states $f_{1}, \cdots, f_{q+i}, i=1, \cdots, L-q_{0}$ need to be calculated only once.

\section{Application to the scattering system $2.6 \mathrm{MeV} \mathrm{Ne}^{9+}$ on $\mathrm{Ne}$}

Detailed double-coincidence measurements between angle-resolved projectile and $K L L$ - or $K L M$-Auger-electrons are available for the collision system $\mathrm{Ne}^{9+}$ on $\mathrm{Ne}$ $[9,10]$. The experiment shows an impact parameter dependent variation of the ratio of Auger-electrons coming from the target $\left(N_{T}\right)$ to the sum of those coming from the projectile and the target $\left(N_{P}+N_{T}\right)$. The $\mathrm{Ne}^{9+}$ projectile bringing one $K$-shell vacancy into the collision system which can be transferred to the Ne target the experimentalists interprete this ratio of $N_{T} /\left(N_{P}+N_{T}\right)$ as a $K$ $K$ charge transfer probability

$P_{K K}=\frac{N_{T}}{\left(N_{T}+N_{P}\right)}$.

We work with this collision system here, using as an effective Hamiltonian the Dirac-Fock-Slater (DFS) single-particle Hamiltonian, and solve the time-dependent single-particle DFS equation

$\left(h_{i}^{\mathrm{DFS}}-\mathrm{i} \hbar \frac{\partial}{\partial t}\right) \psi_{i}(t)=0 \quad$ with

$i=1, \ldots, N$.

The wavefunctions $\psi_{i}(t)$ must satisfy the initial conditions for the $N$ electrons

$$
\begin{aligned}
& \lim _{t \rightarrow-\infty}\left(\psi_{i}(t)-\psi_{i}^{0}(t)\right)=0 \text { with } \\
& i=1, \ldots, N .
\end{aligned}
$$

To solve (14) we expand the time-dependent single-particle wavefunctions $\psi_{i}(t)$ into a set of $M$ molecular wavefunctions $\left\{\varphi^{\mathrm{MO}}\right\}$,

$\psi_{i}(t)=\sum_{m=1}^{M} a_{i m}(t) \varphi_{m}^{\mathrm{MO}}(\mathbf{R}(t)) \mathrm{e}^{-\frac{\mathrm{i}}{\hbar} \int^{j} \varepsilon_{m}\left(\mathbf{R}\left(t^{\prime}\right)\right) \mathrm{d} t^{\prime}}$

with $i=1, \ldots, N$.

In our case the molecular basis states $\varphi^{\mathrm{MO}}$ are solutions from the static self-consistent diatomic Dirac-FockSlater (DFS) equation: [11]

$\bar{h}_{i}^{\mathrm{DFS}}(\mathbf{R}) \varphi_{m}^{\mathrm{MO}}(\mathbf{R})=\varepsilon_{m}(\mathbf{R}) \varphi_{m}^{\mathrm{MO}}(\mathbf{R})$.

with $m=1, \ldots, M$. Inserting ansatz (16) into (14) leads to the equivalent single-particle matrix coupled channel

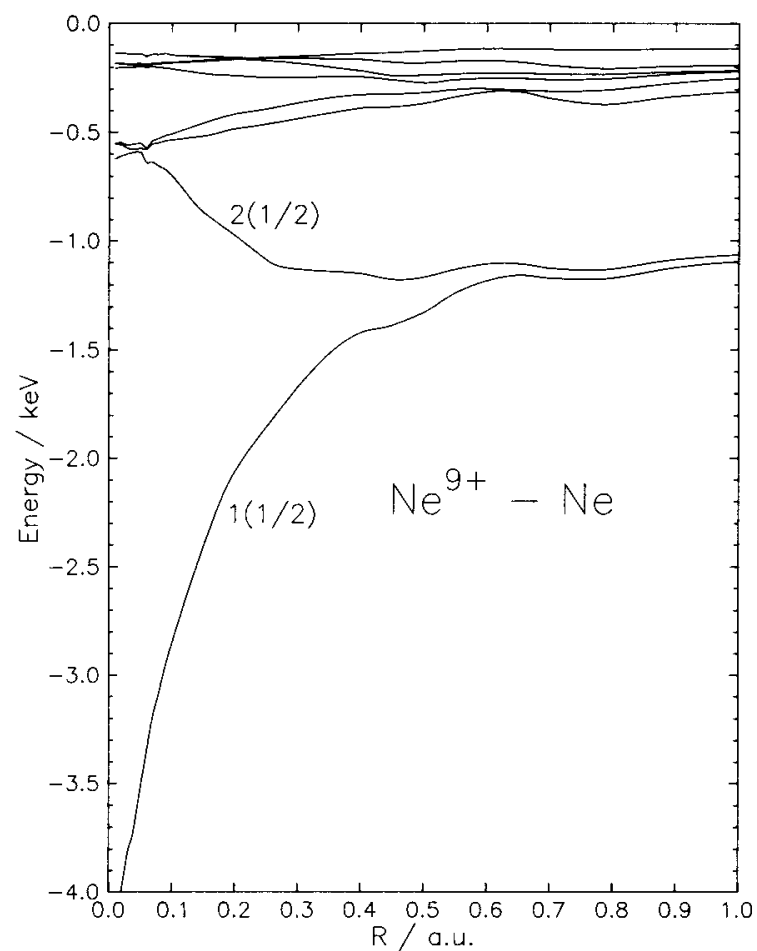

Fig. 1. Correlation diagram for $\mathrm{Ne}^{9+}-\mathrm{Ne}$

equations $[12-14]$

$\mathrm{i} \hbar \frac{\mathrm{d}}{\mathrm{d} t} a_{i l}$

$$
\begin{aligned}
= & \sum_{m=1}^{M} a_{i m}\left\langle\varphi_{l}^{\mathrm{MO}}(R(t))\left|-\mathrm{i} \hbar \frac{\mathrm{d}}{\mathrm{d} t}\right| \varphi_{m}^{\mathrm{MO}}(R(t))\right\rangle \\
& \times \mathrm{e}^{-\frac{\mathrm{i}}{\hbar} \int\left(\varepsilon_{m}\left(\mathbf{R}\left(t^{\prime}\right)\right)-\varepsilon_{l}\left(\mathbf{R}\left(t^{\prime}\right)\right)\right) \mathrm{d} t^{\prime}}
\end{aligned}
$$

with $i=1, \ldots, N$. Solving this single particle coupled channel equation (18) for all electrons involved in the collision system results in $N$ sets of asymptotic single-particle amplitudes $\left\{a_{i j}(t=\infty)\right\}$.

Figure 1 presents the correlation diagram obtained from a large number of static DFS molecular calculations for the system $\mathrm{Ne}^{9+}-\mathrm{Ne}$. The two lowest levels $1(1 / 2)$, and $2(1 / 2)$ can asymptotically $(R \rightarrow+\infty)$ be attributed to $\mathrm{Ne}^{9+} 1 s$-projectile and $\mathrm{Ne} 1 s$-target levels. To allow an exchange with higher levels we solve the coupled-channel equation (18) using the first 20 relativistic oneparticle channels $1(1 / 2) \pm$ to $8(1 / 2) \pm$ and $1(3 / 2) \pm$ to $2(3 / 2) \pm$ presented in Fig. 1 . We chose the initial occupation of these 20 levels according to the asymptotic occupation of the separated atoms at $t=-\infty$. We took all electrons into account obtaining 11 sets of singleparticle amplitudes $\left\{a_{i j}(t=\infty)\right\}$ with $i=1, \cdots, 11$ and $j=1, \cdots, 20$.

We performed two kind of evaluations to compare with the experimental results. In the first evaluation we calculated the probability $P_{K K}$ using a binomial distribution. The second evaluation was done using (12). We 


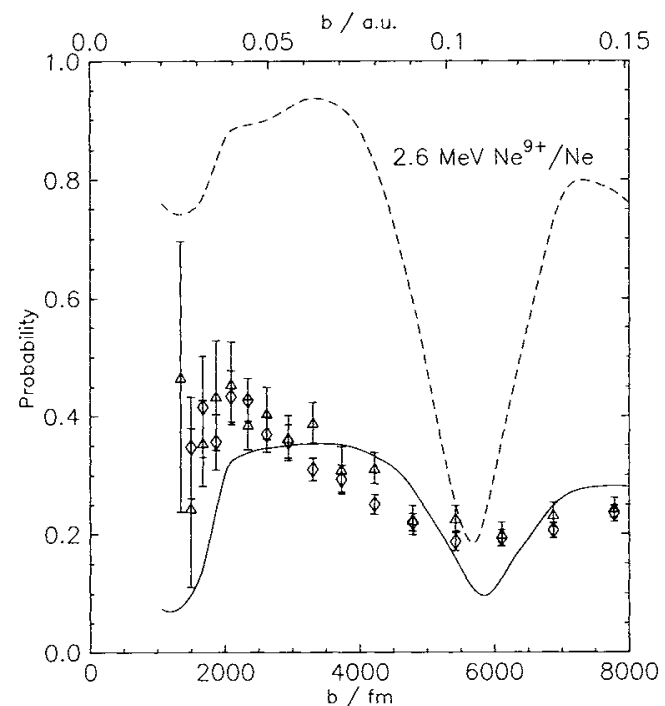

Fig. 2. Exprimental values for $P_{K K}$ charge transfer from Koch et al. $[9,10]$. Dashed curve: $P_{K K}$ using the single-particle model. Full curve: $P_{K K}$ using inclusive probability formalism.

calculated and summed up the impact parameter-dependent many-particle-hole probabilities for each manyparticle state which, after the collision, can lead to $K L L$ Auger electrons from the target or the projectile.

- Single particle model

Within the independent particle model the performance of a 20 channel calculation causes the energetically lowest levels $1(1 / 2)$ and $2(1 / 2)$ (which correspond asymptotically $(t=-\infty)$ to the Neon-projectile $1 s$ shell and Neontarget $1 s$ shell) to experience higher lying $L$ - and $M$-shells via radial and rotational coupling. Therefore, the screening effect of higher shells and its influence on charge transfer is already included in the single particle amplitudes. In the particle picture the probability to find at least one hole in the $K$-shell of the target is given by

$P_{K K}=\left(1-P_{2(1 / 2)}\right)$

where $P_{2(1 / 2)}$ is the probability to find the $2(1 / 2)$ state populated after the collision.

Figure 2 shows the experimental values [9] for the probability $P_{K K}$ versus our theoretical values (dashed curve). Minima and maxima in the oscillatory structure of the experimental measurements are reproduced but the quantitativ agreement with experimental values is poor within the single particle model.

\section{- Many-particle picture via inclusive probabilities}

The experimental $P_{K K}(b)$ charge transfer probability cannot be expressed directly in terms of simple inclusive probabilities. Therefore, as a first step, we calculated the 16 possible final many-particle-hole inclusive probabilities for the 4 lowests levels presented in Table 1. To allow an Auger-decay after the collision we needed additionally at least two electrons in the $L$-shells of the projectile and target. We do not consider many-particle states which
Table 1. 16 possible final states for the four lowest projectile and target levels and number of possible $K L L$-Auger-electrons

\begin{tabular}{|c|c|c|c|c|c|}
\hline \multicolumn{2}{|c|}{ Target } & \multicolumn{2}{|c|}{ Projectile } & \multicolumn{2}{|c|}{$K L L$-Auger } \\
\hline $1 s+$ & $1 s-$ & $1 s+$ & $1 s-$ & Target & Projectile \\
\hline 0 & 0 & 0 & 0 & 2 & 2 \\
\hline 1 & 0 & 0 & 0 & 1 & 2 \\
\hline 0 & 0 & 0 & 1 & 2 & 1 \\
\hline 0 & 0 & 1 & 0 & 2 & 1 \\
\hline 0 & 1 & 0 & 0 & 1 & 2 \\
\hline 1 & 1 & 0 & 0 & 0 & 2 \\
\hline 1 & 0 & 1 & 0 & 1 & 1 \\
\hline 1 & 0 & 0 & 1 & 1 & 1 \\
\hline 0 & 0 & l & I & 2 & 0 \\
\hline 0 & I & 1 & 0 & 1 & 1 \\
\hline 0 & 1 & 0 & 1 & 1 & 1 \\
\hline 1 & 1 & 1 & 0 & 0 & 1 \\
\hline 1 & 0 & 1 & 1 & 1 & 0 \\
\hline 0 & 1 & 1 & 1 & 1 & 0 \\
\hline 1 & 1 & 0 & 1 & 0 & 1 \\
\hline 1 & 1 & 1 & 1 & 0 & 0 \\
\hline
\end{tabular}

could lead to the $K L M$-Auger electrons explicitly because even in the experimental measurements the $K L M$-rate is very small compared to the $K L L$-rate [9].

To match the experimental probability $P_{K K}(b)$ we summed up the 16 probabilities; each of them being multiplied by the factor given in the third resp. fourth column of Table 1. These factors give the number of Auger-electrons from the projectile resp. target for the final manyhole particle state under consideration. This summation leads to the mean number of Auger-electrons $Q_{P}$ resp. $Q_{T}$ available from the projectile resp. target. In analogy to the evaluation of Koch et al. [9] we now calculated the probability $P_{K K}$ as

$$
P_{K K}=\frac{Q_{T}}{\left(Q_{P}+Q_{T}\right)} .
$$

The impact parameter-dependent curve for $P_{K K}$ is shown in Fig. 2 as a full curve. The main shape still compares with the raw $P_{K K}$ charge transfer probability (dashed curve). Additionally, the inclusive many-particle-hole probability gives a good quantitative agreement with experimental data. The comparison of the two theoretical curves shows that a carefull analysis of how the measurements were performed is necessary to answer experimental questions. For the collision system $2.6 \mathrm{MeV} \mathrm{Ne}^{9+}$ on $\mathrm{Ne}$, where Auger-electrons were measured in coincidence with the scattered projectile, it is important to take the $L$-shells of both, projectile and target into account in order to answer experimental questions within the formalism of inclusive probabilities.

This work was in part supported by the Deutsche Forschungsgemeinschaft (DFG) and the Gesellschaft für Schwerionenforschung (GSI). Computer time on CRAY-YMP from the Höchstleistungsrechenzentrum (HLRZ) Jülich is gratefully acknowledged. One of us (HJL) would like to thank for the hospitality during his stay at the University of Kassel. 


\section{References}

1. Reading, J.E.: Phys. Rev. A8, 3262 (1973)

2. Reinhardt, J., Müller, B., Greiner, W., Soff, G.: Phys. Rev. Lett. 43, 1307 (1979)

3. Reading, J.F., Ford, A.F.: Phys. Rev. A21, 124 (1980)

4. Becker, R.L., Ford, A.L., Reading, J.F.: Phys. Rev. A29, 3111 (1984)

5. Lüdde, H.J., Dreizler, R.M.: J. Phys. B18, 107 (1985)

6. Schuch, R., Inwersen, H., Justiniano, E., Schmidt-Böcking, H., Schulz, M., Ziegler, F.: J. Phys. B 17, 2319 (1984)

7. Schulz, M., Justiniano, E., Konrad, J., Schuch, R., Salin, A.: J. Phys. B20, 2057 (1987)
8. Zoran, V., Enulescu, E., Piticu, I., Wintermeyer, G., Kambara, T., Gabr, M., Schuch, R.: Phys. Rev. Lett. 64, 527 (1990)

9. Koch, R.: Ph.D thesis, University of Frankfurt (1991)

10. Koch, R. et al.: to be published

11. Sepp, W.-D., Kolb, D., Sengler, W., Hartung, H., Fricke, B.: Phys. Rev. A33, 3679 (1986)

12. Thies, B., Sepp, W.-D., Fricke, B.: Phys. Lett. A 139, 161 (1989)

13. Kürpick, P., Thies, B., Sepp, W.-D., Fricke, B.: J. Phys. B24, L139 (1991)

14. Kürpick, P., Heinemann, D., Sepp, W.-D., Fricke, B.: Z. Phys. D - Atoms, Molecules and Clusters 22, 407 (1991) 